\title{
Incidence and management of adverse events in patients with relapsed and/or refractory multiple myeloma receiving single-agent carfilzomib
}

This article was published in the following Dove Press journal:

Clinical Pharmacology: Advances and Applications

8 May 2014

Number of times this article has been viewed

\section{R Donald Harvey}

Phase I Clinical Trials Section, Winship Cancer Institute of Emory University, Atlanta, GA, USA
Correspondence: R Donald Harvey Phase I Clinical Trials Section, Winship Cancer Institute of Emory University, I365 Clifton Road NE CPLOI7B, Atlanta, GA 30322, USA

$\mathrm{Tel}+\mathrm{I} 404778$ 438I

Fax +I 4047785520

Email donald.harvey@emory.edu

\begin{abstract}
Carfilzomib, a selective proteasome inhibitor approved in the USA in 2012, is a single agent for relapsed and refractory multiple myeloma. Carfilzomib is administered as a 2-10-minute infusion on days $1,2,8,9,15$, and 16 of a 28 -day cycle at a starting dose of $20 \mathrm{mg} / \mathrm{m}^{2}$ for cycle 1 and a target dose of $27 \mathrm{mg} / \mathrm{m}^{2}$ thereafter. In the pivotal Phase II study (PX-171-003-A1), carfilzomib $20 / 27 \mathrm{mg} / \mathrm{m}^{2}$ provided durable responses in a heavily pretreated population with relapsed and refractory multiple myeloma $(n=266)$, with an overall response rate of $22.9 \%$ and a median duration of response of 7.8 months. In an integrated safety analysis of four Phase II studies, common adverse events (32.7\%-55.5\%) included fatigue, anemia, nausea, thrombocytopenia, dyspnea, and diarrhea. Grade $3 / 4$ adverse events were generally hematologic and included thrombocytopenia (23.4\%), anemia (22.4\%), and lymphopenia (18.1\%). Serious adverse events included pneumonia (9.9\%), acute renal failure (4.2\%), pyrexia $(3.4 \%)$, and congestive heart failure (3.4\%). New or worsening peripheral neuropathy was infrequent $(13.9 \%$ overall, $1.3 \%$ grade 3 , no grade 4 ). This review discusses findings of the integrated safety analysis and provides practical experience from a single institution in managing treatmentrelated and disease-related adverse events. Individualized treatment with proactive management of side effects and complications allows patients with advanced multiple myeloma to remain on carfilzomib for extended periods.
\end{abstract}

Keywords: carfilzomib, relapsed, refractory, myeloma, safety, adverse events, toxicity

\section{Introduction}

Multiple myeloma (MM), the second most common hematologic cancer, is characterized by uncontrolled clonal proliferation of malignant plasma cells within the bone marrow, with associated monoclonal immunoglobulin and protein fragments in blood and urine. Patients often present with hypercalcemia, renal insufficiency, anemia, and/or bone lesions (mnemonically referred to as CRAB), and frequently experience hyperviscosity, fractures, fatigue, and recurrent infections, the leading cause of death in MM, particularly pneumonia. ${ }^{1-3}$ The introduction of targeted therapies, including proteasome inhibitors and immunomodulatory drugs, over the past decade has improved outcomes and survival, ${ }^{4-6}$ but nearly all patients relapse and die from progression of the disease.

At the time of disease progression, patients have usually received multiple treatments, and frequently experience cumulative toxicities, including myelosuppression, cardiac toxicities, and peripheral neuropathy. ${ }^{7}$ Pulmonary and cardiac comorbidities are common ${ }^{8}$ and may be exacerbated by chronic anemia and anti-MM therapies, ${ }^{9}$ 
and patients with relapsed and refractory MM (RRMM) are predisposed to pulmonary infections. ${ }^{2}$

Carfilzomib (Kyprolis ${ }^{\circledR}$, Onyx Pharmaceuticals, Inc., South San Francisco, CA, USA) is a selective proteasome inhibitor that received approval in the USA in 2012 for the treatment of patients with RRMM who have received at least two prior therapies (including bortezomib and an immunomodulatory agent) and have disease progression on or within 60 days of completion of the last therapy. ${ }^{10}$ In the pivotal Phase II study (PX-171-003-A1), single-agent treatment with carfilzomib resulted in an overall response rate of $22.9 \%$ and a median duration of response of 7.8 months. ${ }^{11}$ It was well tolerated, with low rates of dose reductions and discontinuations due to adverse events (AEs).

Recently, an integrated safety analysis for the four Phase II studies of carfilzomib in patients with RRMM was performed to better characterize the safety profile of carfilzomib. ${ }^{12}$ This review highlights the results of the integrated safety analysis and provides practical recommendations for preventing and managing AEs in order to maintain dose intensity, prolong treatment duration, and support quality of life, including recommendations from a large myeloma center at the Winship Cancer Institute of Emory University that participated in these trials.

\section{Phase II studies: design and carfilzomib dosing}

Table $1^{11-16}$ provides a brief overview of the study design and dosing schema for the four Phase II clinical trials included in the integrated safety analysis: PX-171-003-A0
(NCT00511238), ${ }^{13,17}$ PX-171-003-A1 (NCT00511238), ${ }^{11}$ PX-171-004 (NCT00530816), ${ }^{14,15,18}$ and PX-171-005 (NCT00721734). ${ }^{16,19}$ Similarities and differences among these studies are worth noting. The 003-A0 and pivotal 003-A1 studies required refractory MM and prior exposure to bortezomib and immunomodulatory drugs, while the others did not. The 005 study investigated the use of carfilzomib in patients with varying levels of renal impairment, including patients on chronic hemodialysis, while the other studies required patients to have a creatinine clearance $\geq 30 \mathrm{~mL}$ per minute.

Across the Phase II studies, patients received single-agent carfilzomib at doses ranging from 15 to $27 \mathrm{mg} / \mathrm{m}^{2}$ on a 28 -day cycle. At the approved dose, carfilzomib is administered via a 2-10-minute infusion on days 1, 2, 8, 9, 15, and 16 of a 28 -day cycle. During cycle 1 , patients receive a starting dose of $20 \mathrm{mg} / \mathrm{m}^{2}$, and if tolerated, the dose can be increased to a target of $27 \mathrm{mg} / \mathrm{m}^{2}$ per infusion during cycle 2 and beyond. ${ }^{10}$ Subtherapeutic dexamethasone $(4 \mathrm{mg})$ can and should be administered prior to carfilzomib during cycle 1 to reduce infusion reactions and in subsequent cycles as indicated.

\section{Patient population in Phase II studies}

In the population included in the integrated safety analysis, patients were predominantly Caucasian (72.4\%) and male (57.4\%), with a median age of 64 (37-87) years and a median time since diagnosis of $4.8(0.5-24.4)$ years..$^{12}$ The distribution of European Cooperative Oncology Group

Table I Overview of Phase II safety study designs ${ }^{11-16}$ NCT00511238 ${ }^{17}$

\section{PX-171-003-A0}

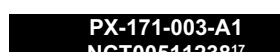
NCT00511238 ${ }^{17}$

\begin{tabular}{|l|l|}
\hline Key eligibility criteria & - Relapsed and refractory \\
- $\geq 2$ prior regimens \\
- Responded to $\geq 1$ regimen \\
- Refractory to most recent \\
- Prior tx with bortezomib, \\
IMiD, anthracycline, and \\
alkylating agents
\end{tabular}
Refractory to most recent

- Relapsed and refractory
- $\geq 2$ prior regimens
- Responded to $\geq 1$ regimen
- Refractory to most recent
- Prior tx with bortezomib,
IMiD, anthracycline, and
alkylating agents
- ECOG PS 0-2
- No significant CVD
- No grade $3 / 4$ PN or grade
2 with pain
$\geq 50,000 / \mathrm{mm}^{3}$
$\geq 8 \mathrm{~g} / \mathrm{dL}^{3}$
$\geq 1,000 / \mathrm{mm}^{3}$
$\quad 3 \times \mathrm{ULN}$
$>30 \mathrm{~mL} / \mathrm{min}^{2}$
$20 / 27 \mathrm{mg} / \mathrm{m}^{2 \mathrm{c}}$
$12 \mathrm{cycles}$

- Relapsed and refractory - Prior tx with bortezomib, IMiD, anthracycline, and alkylating agents No significant CVD o grade $3 / 4 \mathrm{PN}$ or grad $\geq 50,000 / \mathrm{mm}^{3}$ $\geq 8 \mathrm{~g} / \mathrm{dL}$ $\geq 1,000 / \mathrm{mm}$ $<3 \times$ ULN 12 cycles

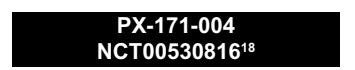
NCT00530816

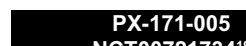
NCT00721734 19

\begin{tabular}{|c|c|}
\hline $\begin{array}{l}\text { - Relapsed and/or refractory } \\
\text { - } 1-3 \text { prior regimens } \\
\text { - Responded to 1st-line } \\
\text { regimen }\end{array}$ & $\begin{array}{l}\text { - Relapsed, refractory, and/or } \\
\text { progressive } \\
\text { - } \geq 2 \text { prior regimens } \\
\text { - Responded to } \geq 1 \text { regimen }\end{array}$ \\
\hline $\begin{array}{l}\text { - ECOG PS 0-2 } \\
\text { - No significant CVD } \\
\text { - No grade } 3 / 4 \text { PN or grade } 2 \\
\text { with pain }\end{array}$ & $\begin{array}{l}\text { - ECOG PS 0-2 } \\
\text { - No significant CVD } \\
\text { - No grade } 3 / 4 \text { PN or grade } 2 \\
\text { with pain }\end{array}$ \\
\hline 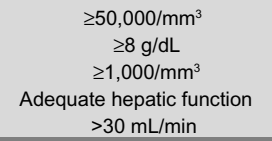 & $\begin{array}{l}\qquad \begin{array}{c}\geq 30,000 / \mathrm{mm}^{3} \\
\quad \geq 7 \mathrm{~g} / \mathrm{dL} \\
\geq 1,000 / \mathrm{mm}^{3}\end{array} \\
\text { Adequate hepatic function } \\
\text { Various levels of RI }\end{array}$ \\
\hline $\begin{array}{c}20 \text { or } 20 / 27 \mathrm{mg} / \mathrm{m}^{2 \mathrm{c}} \\
12 \text { cycles }\end{array}$ & $\begin{array}{c}15 / 20 / 27 \mathrm{mg} / \mathrm{m}^{2 \mathrm{~d}} \\
12 \text { cycles }\end{array}$ \\
\hline
\end{tabular}

Notes: ${ }^{a} \mathrm{New}$ York Heart Association Class III-IV heart failure or recent myocardial infarction/unstable angina were excluded; badministered as a 2-10 min intravenous infusion on days I, 2, 8, 9, I5, and 16 of a 28 -day cycle; ' starting dose of $20 \mathrm{mg} / \mathrm{m}^{2}$ in cycle I, target dose of $27 \mathrm{mg} / \mathrm{m}^{2}$ thereafter; ${ }^{\mathrm{d}} \mathrm{starting}$ dose $15 \mathrm{mg} / \mathrm{m}^{2}$ for cycle I, $20 \mathrm{mg} / \mathrm{m}^{2}$ for cycle 2 with target dose of $27 \mathrm{mg} / \mathrm{m}^{2}$ thereafter.

Abbreviations: ALT, alanine aminotransferase; ANC, absolute neutrophil count; AST, aspartate aminotransferase; $\mathrm{CrCl}$, creatinine clearance; CVD, cardiovascular disease; ECOG, Eastern Cooperative Oncology Group; Hb, hemoglobin; IMID, immunomodulatory drug; min, minute; PN, peripheral neuropathy; PS, performance status; RI, renal insufficiency; ULN, upper limit of normal. 
performance status was $29.7 \%, 58.4 \%$, and $11.6 \%$ for scores of 0,1 , and 2 , respectively. Patients were heavily pretreated with a median of four prior regimens, with many refractory to lenalidomide $(69.6 \%)$, bortezomib (51.7\%), and thalidomide (39.7\%). At study entry, patients had a number of comorbidities and disease-related and treatment-related complications, the most common being hematologic, including anemia (89.3\%), lymphopenia (65.4\%), neutropenia (64.4\%), and thrombocytopenia (37.4\%). ${ }^{20}$ Additionally, $73.6 \%$ of patients had a history of cardiovascular events, and $70.0 \%$ were receiving one or more cardiac medications at study entry. Most patients $(84.8 \%)$ had a history of peripheral neuropathy. ${ }^{12}$ Across the four Phase II studies, $23.8 \%$ of patients had moderate to severe renal dysfunction (creatinine clearance $<50 \mathrm{~mL}$ per minute), and $39.4 \%$ had mild renal dysfunction (creatinine clearance 50 to $<80 \mathrm{~mL}$ per minute).

\section{Overall analysis \\ Safety and tolerability of carfilzomib}

The integrated safety analysis further defines the safety and tolerability of single-agent carfilzomib. The median number of cycles given was four (range 1-21), with $19.0 \%$ of patients starting $\geq 12$ cycles and $51.0 \%$ discontinuing treatment prior to 12 cycles due to disease progression; $14.6 \%$ required a dose reduction due to an $\mathrm{AE}$ and $14.8 \%$ discontinued treatment due to an AE (Table 2). ${ }^{12}$

Table $3^{12,21}$ summarizes AEs that emerged during treatment, and Table $4^{12}$ provides rates for AEs grouped by organ system, including cardiac, pulmonary, and renal. The most common AEs overall included fatigue, anemia, nausea, dyspnea, and diarrhea (range 32.7\%-55.5\%). ${ }^{12}$ AEs of grade $\geq 3$ in severity were generally hematologic, and included thrombocytopenia, anemia, lymphopenia, and neutropenia (range 10.3\%-23.4\%). Nonhematologic
AEs associated with carfilzomib were generally grade $1 / 2$ in severity, although serious AEs did include pneumonia $(9.9 \%)$, acute renal failure $(4.2 \%)$, pyrexia $(3.4 \%)$, and congestive heart failure $(3.4 \%)$.

Overall, there were 37 deaths on study or within 30 days of the last dose. Of these, seven were the result of AEs deemed at least possibly related to carfilzomib, including cardiac arrest $(n=2)$, hepatic failure $(n=1)$, dyspnea $(n=1)$, multiorgan failure $(n=1)$, cardiac disease $(n=1)$, and death from unknown cause $(n=1) .{ }^{12}$

The following sections will discuss AEs of clinical interest in more depth, along with approaches to mitigate their incidence and severity in order for patients to maintain carfilzomib treatment and dose intensity (Table $5^{10,22,23}$ ).

\section{Hematologic AEs}

Among AEs considered related to carfilzomib treatment, hematologic events were generally the most common, with rates of $28.3 \%$ for thrombocytopenia, $26.8 \%$ for anemia, $17.7 \%$ for lymphopenia, and $16.0 \%$ for neutropenia. ${ }^{12,20}$ Generally, these events were transient and manageable, and serious clinical sequelae, such as fever and bleeding events, were rare. In general, patients receiving carfilzomib should be closely monitored with a complete blood count before each dose in addition to standard supportive care, as discussed in the following subsections.

\section{Thrombocytopenia}

Thrombocytopenia was transient and cyclic, with platelets predictably decreasing to a nadir by day 8 of the 28 -day cycle and returning to normal by the first day of the subsequent cycle. ${ }^{12,20}$ No clinically significant bleeding events associated with thrombocytopenia or cumulative thrombocytopenia were reported. Decrease in platelet count was generally managed with platelet transfusions as per institutional

Table 2 Patient disposition

\begin{tabular}{|c|c|c|c|c|c|}
\hline PX-|7| & $\begin{array}{l}003-A 0 \\
n=46\end{array}$ & $\begin{array}{l}003-A \mid \\
n=266\end{array}$ & $\begin{array}{l}004 \\
n=164\end{array}$ & $\begin{array}{l}005 \\
n=50\end{array}$ & $\begin{array}{l}\text { ISA } \\
n=526\end{array}$ \\
\hline Median cycles ( $n$ ) & 3 & 4 & 6 & 4 & 4 \\
\hline Dose reduced due to $\mathrm{AE}, \mathrm{n}(\%)$ & $3(6.5)$ & $47(17.7)$ & $18(11.0)$ & $9(18.0)$ & $77(14.6)$ \\
\hline$\geq 12$ cycles or on therapy, $n(\%)$ & $4(8.7)$ & $40(15.0)$ & $55(33.5)$ & $16(32.0)$ & $115(21.9)$ \\
\hline$<12$ cycles, $\mathrm{n}(\%)$ & $42(91.3)$ & $226(85.0)$ & $109(66.5)$ & $34(68.0)$ & $4 I I(78 . I)$ \\
\hline Progressive disease & $23(50.0)$ & $157(59.0)$ & $64(39.0)$ & $24(48.0)$ & $268(51.0)$ \\
\hline $\mathrm{AE}$ & $13(28.3)$ & $33(12.4)$ & $26(15.9)$ & $6(12.0)$ & $78(14.8)$ \\
\hline Withdrew consent & $2(4.3)$ & $22(8.3)$ & $9(5.5)$ & $4(8.0)$ & $37(7.0)$ \\
\hline Other & $4(8.7)$ & $14(5.3)$ & $10(6.1)$ & $0(0)$ & $28(5.3)$ \\
\hline
\end{tabular}

Notes: *At data cutoff. Obtained from Haematologica/the Hematology Journal website http://www.haematologica.org. ${ }^{12}$ Abbreviations: $\mathrm{AE}$, adverse event; ISA, integrated safety analysis. 
Table 3 Integrated analysis of adverse events from four Phase II studies of single-agent carfilzomib in patients with relapsing and remitting multiple myeloma $(n=526)$

\begin{tabular}{|c|c|c|c|c|}
\hline & $\begin{array}{l}\text { All grades } \\
\text { n (\%) }\end{array}$ & $\begin{array}{l}\text { All grades related } \\
\text { n (\%) }\end{array}$ & $\begin{array}{l}\text { Grades 3/4 } \\
\mathrm{n}(\%)\end{array}$ & $\begin{array}{l}\text { SAE } \\
\text { n (\%) }\end{array}$ \\
\hline \multicolumn{5}{|l|}{ Hematologic } \\
\hline Anemia & $246(46.8)$ & |4| (26.8) & I I 8 (22.4) & $7(1.3)$ \\
\hline Thrombocytopenia & $191(36.3)$ & $149(28.3)$ & $123(23.4)$ & $6(1.1)$ \\
\hline Lymphopenia & $126(24.0)$ & $93(17.7)$ & $95(18.1)$ & 0 \\
\hline Neutropenia & $109(20.7)$ & $84(16.0)$ & $54(10.3)$ & $2(0.4)$ \\
\hline Leukopenia & $71(13.5)$ & $56(10.6)$ & $28(5.3)$ & 0 \\
\hline \multicolumn{5}{|l|}{ Nonhematologic } \\
\hline Fatigue & $292(55.5)$ & $218(41.4)$ & $40(7.6)$ & 0 \\
\hline Nausea & $236(44.9)$ & $185(35.2)$ & $7(1.3)$ & 0 \\
\hline Dyspnea & $182(34.6)$ & $107(20.3)$ & $26(4.9)$ & II (2.I) \\
\hline Diarrhea & $172(32.7)$ & $118(22.4)$ & $5(1.0)$ & $3(0.6)$ \\
\hline Pyrexia & $160(30.4)$ & $79(15.0)$ & $9(1.7)$ & $18(3.4)$ \\
\hline Upper respiratory tract infection & $149(28.3)$ & $38(7.2)$ & $17(3.2)$ & $5(1.0)$ \\
\hline Headache & $145(27.6)$ & $83(15.8)$ & $7(1.3)$ & 0 \\
\hline Cough & $137(26.0)$ & $39(7.4)$ & $\mathrm{I}(0.2)$ & $\mathrm{I}(0.2)$ \\
\hline Increased serum creatinine & $127(24.1)$ & $93(17.7)$ & $14(2.7)$ & $7(1.3)$ \\
\hline Peripheral edema & $126(24.0)$ & $56(10.6)$ & $3(0.6)$ & 0 \\
\hline Vomiting & $117(22.2)$ & $85(16.2)$ & $5(1.0)$ & $2(0.4)$ \\
\hline Constipation & $110(20.9)$ & $57(10.8)$ & $\mathrm{I}(0.2)$ & 0 \\
\hline Back pain & $106(20.2)$ & $12(2.3)$ & $15(2.9)$ & $\mathrm{I}(0.2)$ \\
\hline Pneumonia $^{c}$ & 67 (12.7) & $24(4.6)$ & $55(10.5)$ & $52(9.9)$ \\
\hline
\end{tabular}

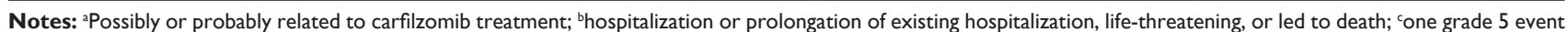
in study 003-AI. Adverse events graded according to the National Cancer Institute Common Terminology Criteria. ${ }^{21}$ Obtained from Haematologica/the Hematology Journal website http://www.haematologica.org. ${ }^{12}$

Abbreviation: SAE, serious AEs.

guidelines, and, rarely, dose reduction. Dose reduction and discontinuation rates due to thrombocytopenic AEs were low ( $1.1 \%$ and $1.0 \%$, respectively).

In clinical practice, carfilzomib should be withheld for grade 4 thrombocytopenia, ${ }^{10}$ and platelet transfusion

Table 4 Integrated analysis of AE by grouped-term organ system from four Phase II studies of single-agent carfilzomib in patients with relapsing and remitting multiple myeloma $(n=526)$

\begin{tabular}{llll}
\hline & $\begin{array}{l}\text { Any AE } \\
\text { n (\%) }\end{array}$ & $\begin{array}{l}\geq \text { grade 3 } \\
\text { n (\%) }\end{array}$ & $\begin{array}{l}\text { SAE } \\
\text { n (\%) }\end{array}$ \\
\hline Any cardiac & $116(22.1)$ & $50(9.5)$ & $4 I(7.8)$ \\
Cardiac arrhythmia & $70(13.3)$ & $12(2.3)$ & $1 \mathrm{II}(2.1)$ \\
Cardiac failure & $38(7.2)$ & $30(5.7)$ & $26(4.9)$ \\
Ischemic heart disease & $18(3.4)$ & $7(1.3)$ & $5(1.0)$ \\
Cardiomyopathy & $9(1.7)$ & $3(0.6)$ & $2(0.4)$ \\
Any respiratory & $363(69.0)$ & $54(10.3)$ & $34(6.5)$ \\
Dyspnea & $222(42.2)$ & $26(4.9)$ & $1 \mathrm{II}(2.1)$ \\
Cough & $137(26.0)$ & $1(0.2)$ & $1(0.2)$ \\
Pneumonia & $67(12.7)$ & $55(10.5)$ & $52(9.9)$ \\
Any grouped renal impairment & $174(33.1)$ & $38(7.2)$ & $32(6.1)$ \\
Increased serum creatinine & $127(24.1)$ & $14(2.7)$ & $7(1.3)$ \\
Acute renal failure & $28(5.3)$ & $23(4.4)$ & $22(4.2)$ \\
Renal failure & $20(3.8)$ & $6(1.1)$ & $7(1.3)$ \\
\hline
\end{tabular}

Notes: Obtained from Haematologica/the Hematology Journal website http://www. haematologica.org. ${ }^{12}$

Abbreviations: AE, adverse event; SAE, serious adverse event. should be considered as indicated (eg, active bleeding, asymptomatic platelet count $<10,000 / \mathrm{mm}^{3}$ ). The etiology of thrombocytopenia should also be considered, as its presentation may be related to disease where aggressive therapy may lead to improved platelet counts. At Emory, we treat patients with platelet counts $>25,000 / \mathrm{mm}^{3}$, recognizing the elasticity of platelet recovery following treatment. The carfilzomib treatment schedule allows for frequent assessment of blood counts, and therefore a greater understanding of an individual's nadir and peak platelet counts.

\section{Neutropenia}

Neutrophil counts have also been shown to be cyclic with carfilzomib treatment, reaching a nadir at day 15 , and returning to normal by day 1 of the next cycle. ${ }^{20}$ In the integrated safety analysis, febrile neutropenia occurred infrequently $(1.1 \%)$, dose reduction and discontinuation rates due to neutropenia were very low $(1.1 \%$ and $0.2 \%$, respectively), and a shift from grade 0 (absence) neutropenia at baseline to grade $3 / 4$ was uncommon (7/187 patients, $3.7 \%) .^{12}$

Carfilzomib dose reductions may be used to manage neutropenia. Similar to the management of grade 4 
Table 5 Clinical practice recommendations for carfilzomib ${ }^{10,22,23}$

Emory approach

\section{Prophylaxis}

Hydration

- Reduces the risk of renal toxicity and TLS

Subtherapeutic dexamethasone

- Prevents or alleviates flu-like infusion symptoms

Allopurinol

- Reduces the risk of renal toxicity and TLS

Antivirals (eg, acyclovir, famciclovir, valaciclovir)

- Prevents herpes virus infections Antibacterials (eg, ciprofloxacin, cotrimoxazole, levofloxacin, moxifloxacin)

- Prevents infections related to immunosuppression, particularly in patients at risk of certain infections

\section{Management of AEs}

Hematologic toxicity Neutropenia (grade 3/4)

Thrombocytopenia (grade 4)

Cardiac toxicity

Grade 3/4 cardiac toxicity;

new onset or worsening of congestive heart failure, decreased left ventricular function, or myocardial ischemia Pulmonary hypertension or

Peripheral neuropathy (grade 3/4)

Pulmonary complications (grade 3/4)

or

Other grade $3 / 4$

nonhematologic toxicities

Hepatic toxicity

Grade 3/4 elevation of transaminases, bilirubin, or other liver abnormalities
- Instruct the patient to drink 8 cups of water a day during dosing

- 250-500 mL of normal saline should be administered before and after infusion (as needed)

- $4 \mathrm{mg}$ (PO or IV) should be administered before infusion during Cycle I with the $20 \mathrm{mg} / \mathrm{m}^{2}$ starting dose and before all doses during the first cycle of the target dose of $27 \mathrm{mg} / \mathrm{m}^{2}$

- 100-300 mg PO daily

- Antiviral agent should be prescribed in patients at risk

- Common regimen is acyclovir $400 \mathrm{mg}$ PO BID

- Appropriate antibacterial agent should be prescribed in patients at risk

- Withhold the dose of carfilzomib

- If the patient fully recovers before the next scheduled dose, continue at same dose level

- Thrombocytopenia: if the patient recovers to grade 3 thrombocytopenia, reduce dose by one dose level

- Neutropenia: if the patient recovers to grade 2 neutropenia, reduce the dose by one dose level ${ }^{2}$

- If tolerated, the reduced dose may be escalated to the previous dose at the discretion of the physician

- Withhold the dose of carfilzomib until the event resolves or returns to baseline

- After resolution, consider whether the patient should restart at a reduced dose $\mathrm{a}^{\mathrm{a}}$

- If reduced dose is tolerated, the dose may be escalated to the previous dose at the discretion of the physician

- Withhold the dose of carfilzomib until the event resolves or returns to baseline

- Restart at the dose used before the event or reduce dose $\mathrm{e}^{\mathrm{a}}$ at the discretion of the physician

- If reduced dose is tolerated, the dose may be escalated to the previous dose at the discretion of the physician

- Withhold the dose of carfilzomib until the event resolves or returns to baseline

- Consider restarting at the next scheduled treatment with one dose level reduction

- If reduced dose is tolerated, the dose may be escalated to the previous dose at the discretion of the physician

- Withhold the dose of carfilzomib until the event resolves or returns to baseline

- After resolution, consider if restarting carfilzomib is appropriate

- If appropriate, reinitiate at a reduced dose $e^{a}$ with frequent monitoring of liver function

- If reduced dose is tolerated, the dose may be escalated to the previous dose at the discretion of the physician
- Use $500 \mathrm{~mL}$ normal saline before and after infusion, except in patients at risk for fluid overload (renal, cardiac) where $250 \mathrm{~mL}$ volumes are employed

- Use $4 \mathrm{mg}$ before the dose for all infusions except when contraindicated

- Used selectively in patients with high tumor burden (eg, elevated LDH, uric acid, $>50 \%$ plasma cells on bone marrow examination)

- Acyclovir 400 mg PO BID in all patients

- Co-trimoxazole double strength PO daily MWF in all patients except when contraindicated

- Assess drug versus disease causes

- Hold carfilzomib if ANC $<1,000 / \mathrm{mm}^{3}$, platelets $<25,000 / \mathrm{mm}^{3}$

- Per product information

- Per product information

- Per product information

- Hold for AST/ALT $>5$ times the ULN and bilirubin $>3$ times ULN

- Assess other potential causes

- Reinitiate following resolution per product information 
Table 5 (Continued)

\begin{tabular}{lll}
\hline & & Emory approach \\
\hline Renal toxicity & - Withhold the dose of carfilzomib until renal function & Assess cause and timing of renal insult \\
Serum creatinine $\geq 2 \times$ baseline & recovers to grade I or to baseline; monitor renal function & - If stabilized, clearly due to myeloma, \\
- If renal dysfunction is attributable to carfilzomib, & restart the next scheduled treatment at a reduced dose \\
- If renal dysfunction is not attributable to carfilzomib, & restart at the dose used before the event \\
- If reduced dose is tolerated, the dose may be escalated & to the previous dose at the discretion of the physician
\end{tabular}

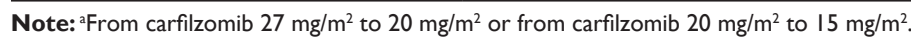

Abbreviations: AE, adverse event; ALT, alanine aminotransferase; ANC, absolute neutrophil count; AST, aspartate aminotransferase; BID, twice daily; IV, intravenously; LDH, lactate dehydrogenase; MWF, Monday, Wednesday, Friday; PO, orally; TLS, tumor lysis syndrome; ULN, upper limit of normal.

thrombocytopenia, carfilzomib should be withheld for patients experiencing grade $3 / 4$ neutropenia. ${ }^{10}$ Granulocyte colony-stimulating factor products are also an option and should be used prophylactically in patients at high risk for febrile neutropenia. ${ }^{24-26}$ If neutrophil recovery is incomplete or slower than predicted and/or other lineages are affected, differentiation of etiology of neutropenia (drug versus disease) through bone marrow evaluation may be required.

\section{Anemia}

Hemoglobin remained stable throughout treatment, and the mean and median nadirs remained at grade 1 in the clinical trials. ${ }^{12,20}$ Dose reduction and discontinuation rates due to anemia were very low ( $0.4 \%$ and $0.6 \%$, respectively). Patients should have hemoglobin levels monitored routinely; the dose of carfilzomib does not need to be withheld or modified for anemia that can be managed with standard measures, including red blood cell transfusion when indicated..$^{10}$ At Emory, these indications include increasing fatigue, shortness of breath, and/or tachycardia with low hemoglobin levels $(\leq 8 \mathrm{~g} / \mathrm{dL})$.

\section{Nonhematologic AEs}

\section{Infusion reactions and acute effects}

Events within the first 24-48 hours of treatment with carfilzomib include infusion-related reactions, increases in certain laboratory values (ie, creatinine, transaminases), and increased blood pressure. These can occur immediately following or up to 24 hours after administration of carfilzomib. First-dose effects associated with carfilzomib refer to a constellation of symptoms, including fever, chills, and rigors. Infusion-related reactions were characterized by a spectrum of flu-like symptoms, including fever, rigor, chills, arthralgia, myalgia, facial flushing, facial edema, vomiting, weakness, dyspnea, hypotension, syncope, chest tightness, and angina. The incidence of infusion-related reactions for various symptoms occurring within the first day of dosing in cycle 1 were dyspnea (12\%), fever (8\%), chills $(4 \%)$, arthralgia (3\%), myalgia (2\%), and flushing (1\%) (data on file, Onyx Pharmaceuticals, Inc., 2013).

Many of the frequently reported AEs associated with carfilzomib, especially those associated with infusion-related flu-like symptoms, may be prevented or alleviated with proper prophylaxis. ${ }^{10}$ Patients should receive subtherapeutic dexamethasone $(4 \mathrm{mg})$ before administration of carfilzomib during cycle 1 with the starting dose of $20 \mathrm{mg} / \mathrm{m}^{2}$, and before all doses during the first cycle of the $27 \mathrm{mg} / \mathrm{m}^{2}$ target dose. Prophylaxis with dexamethasone should be reinstated if symptoms develop or reappear during subsequent cycles. Prior to treatment, patients should be educated about the symptoms associated with infusion reactions and instructed to report them should they occur.

\section{Cardiac AEs}

In assessing cardiac AEs in heavily pretreated patients with $\mathrm{MM}$, it is important to be mindful of the prevalence of cardiovascular disease at baseline due to age-related and disease-related risks, including exposure to treatments associated with cardiotoxicity, such as anthracyclines, alkylators, proteasome inhibitors, and stem cell transplant. ${ }^{27-30} \mathrm{In}$ the four Phase II studies, $73.6 \%$ of patients had a history of cardiovascular events and $70.0 \%$ were receiving a cardiovascular or diabetic medication, although it should be noted that significant cardiovascular disease was an exclusion criterion in these studies (Table 1). Prior treatments in the integrated safety analysis population included bortezomib (75.5\%) and anthracyclines $(52.5 \%))^{12,31}$

Cardiac failure events (eg, congestive heart failure, pulmonary edema, decreased left ventricular function) were reported in $7.2 \%$ of patients, and the most common cardiac AEs were arrhythmias; most were low-grade, benign, supraventricular events (ie, tachycardia and palpitations). ${ }^{12,31}$ 
Cardiac AEs grade $\geq 3$ included cardiac failure (5.7\%), cardiac arrhythmia $(2.3 \%)$, ischemic heart disease $(1.3 \%)$, and cardiomyopathy $(0.6 \%)$. The rate of on-study cardiac events did not increase over time. Cardiac AEs resulting in treatment discontinuation ( $4.4 \%$ overall) included congestive heart failure (1.5\%), cardiac arrest $(1.0 \%)$, and myocardial ischemia $(0.6 \%)$.

Patients receiving carfilzomib should be closely monitored for new or worsening cardiac symptoms, and any symptoms should be managed promptly. ${ }^{10}$ For those experiencing new or worsening congestive heart failure symptoms, decreased left ventricular function, or myocardial ischemia, carfilzomib treatment should be withheld until resolution or return to baseline, with subsequent treatment decisions based on careful risk/benefit assessment. At Emory, we have elected to treat patients with proteasome inhibitor-sensitive disease and New York Heart Association Class I-II heart failure with no more than $250 \mathrm{~mL}$ of hydration before and after each carfilzomib dose, with close monitoring.

\section{Pulmonary AEs}

Dyspnea was reported as a grouped term in $42.2 \%$ of patients treated with carfilzomib enrolled in the four Phase II clinical trials (Table 4). ${ }^{12,31}$ Most events were mild (grade 1 or 2), transient, occurred within one day of dosing, and resolved without dose reduction or discontinuation. Grade 3 dyspnea was reported by $4.8 \%$ of patients; there were no grade 4 events, and one death occurred in the setting of concurrent congestive heart failure. Other pulmonary AEs of clinical importance included pleural effusion in $4 \%$ of patients and pulmonary arterial hypertension in $2 \%$ of patients. ${ }^{10,12}$ Grade 3 pulmonary arterial hypertension was reported in $<1 \%$ of patients and in three patients was considered possibly related to carfilzomib. No dose reductions due to pulmonary arterial hypertension were reported, but one patient discontinued carfilzomib due to pulmonary arterial hypertension. Cardiopulmonary evaluation (eg, cardiac imaging) to determine the underlying etiology is recommended for patients presenting with signs or symptoms of persistent dyspnea and/or pulmonary arterial hypertension. ${ }^{10}$ For grade $\geq 3$ pulmonary events, treatment should be withheld until signs and symptoms resolve or return to baseline. In our experience, dyspnea seen following carfilzomib is self-limiting, and requires no intervention other than patient education. For prolonged episodes, referral to pulmonologists and/or cardiologists may be necessary based on the constellation of symptoms.

Across the four studies, respiratory infections were reported in $18.8 \%$ of patients. Pneumonia was the most common respiratory $\mathrm{AE}(12.7 \%)$ and the most common serious AE (9.9\%). ${ }^{12,31}$ Respiratory infections contributed to the death of two patients on carfilzomib, but neither death was attributed to carfilzomib. A small percentage of patients who experienced pneumonia had dose reduction $(0.4 \%)$ or discontinued therapy (1.9\%).

Prophylaxis with antibacterials can be considered for patients at high risk for infections, including those with recurrences of sinusitis, pneumonia, or urinary tract infections, and patients with decreased immunoglobulins (other than the monoclonal protein) for prolonged periods may be at risk for recurrent infections. At Emory, we consider supplemental intravenous immune globulin for patients with two or more serious infections (eg, requiring hospitalization or two different antimicrobial regimens to resolve).

\section{Renal AEs}

In the 005 study, the pharmacokinetics and safety of carfilzomib were evaluated in patients with normal renal function and those with mild, moderate, and severe renal impairment, including patients on chronic hemodialysis. ${ }^{16}$ Carfilzomib disposition and $\mathrm{AE}$ rate and severity in patients with various degrees of renal insufficiency were similar to patients without renal impairment, demonstrating the utility of carfilzomib in patients with renal insufficiency. Concurrent use of nephrotoxic agents such as bisphosphonates should be carefully considered in patients with renal insufficiency receiving carfilzomib. At Emory, we prefer to give two or more cycles of carfilzomib prior to use of a bisphosphonate for fracture prevention to assess stability of renal function. We give dose-adjusted zoledronic acid to patients with creatinine clearance as low as $30 \mathrm{~mL}$ per minute or pamidronate $60 \mathrm{mg}$ over $4-6$ hours in patients with creatinine clearance $<30 \mathrm{~mL}$ per minute.

In the integrated safety analysis, renal function did not worsen during the course of carfilzomib treatment in $87 \%$ of patients with post-baseline creatinine values (447/515). ${ }^{12,32}$ Among 68 patients with worsening renal function, the median reduction in estimated glomerular filtration rate was $41.99 \mathrm{~mL}$ per minute from baseline to the first day of worsening within a median time of 44.5 days. A distinction between drug-related and myeloma-related worsening was not possible. In $46 \%$ (31/68) of patients, the effect was transient (ie, serum creatinine resolved to within $20 \%$ of baseline values), with a median duration of 1.4 (0.29-21.1) weeks, and no patients discontinued treatment due to renal dysfunction. In 54\% (37/68) of patients, the result was nontransient and did not resolve as of the last available creatinine 
values, with eight patients discontinuing treatment due to renal dysfunction. Overall, renal AEs resulting in carfilzomib dose reduction or discontinuation were uncommon in the population.

In practice, carfilzomib should be withheld for patients with serum creatinine two or more times the baseline value until renal function has recovered to grade 1 or baseline, with subsequent increased monitoring of renal function. ${ }^{10}$ Clinically, if creatinine increases by two or more times the baseline value, frequently the distinction between myeloma and drug causes is clear, ie, total protein values increase substantially in concert with creatinine, indicating disease progression. Clearance of carfilzomib has not been studied during hemodialysis, so it should be administered after the procedure.

\section{Peripheral neuropathy}

Across the four Phase II studies, carfilzomib was associated with a low rate of mild to moderate, nondose-limiting peripheral neuropathy and did not exacerbate pre-existing peripheral neuropathy. ${ }^{12,33}$ Although the majority of patients (85\%) in the Phase II studies had medical histories of peripheral neuropathy, new or worsening peripheral neuropathy occurred infrequently during treatment with carfilzomib (overall 14\%, 1\% were grade 3 , and no grade 4 peripheral neuropathy). Among patients with active peripheral neuropathy at baseline $(72 \%$ of the population, all grade $1 / 2$ ), $87 \%$ did not report AEs related to peripheral neuropathy at any time during treatment with carfilzomib. Given that most of these peripheral neuropathy events occurred before cycle 6 , there was no evidence of a cumulative peripheral neuropathy toxicity. Overall rates of dose modification and discontinuation due to peripheral neuropathy were low ( $0.8 \%$ and $0.2 \%$, respectively).

If grade $\geq 3$ peripheral neuropathy occurs following administration of carfilzomib, treatment should be withheld until it resolves or returns to baseline. ${ }^{10}$ In our center's experience, patients with peripheral neuropathy of grade 2 with pain prior to initiating carfilzomib very rarely progress to grade 3 after starting treatment; patients who do experience a worsening of peripheral neuropathy usually have substantial prior thalidomide and/or bortezomib exposure, clouding the etiology of peripheral neuropathy. We have also safely treated patients on stable medication regimens for pain associated with peripheral neuropathy (eg, pregabalin, gabapentin) with carfilzomib.

\section{Gastrointestinal AEs}

Gastrointestinal AEs were common, with $72.4 \%$ of patients experiencing at least one gastrointestinal AE; the most common events were nausea (44.9\%), diarrhea $(32.7 \%)$, vomiting (22.2\%), and constipation $(20.9 \%){ }^{10,12}$ The majority of gastrointestinal events were grade $1 / 2$ and only $2.9 \%$ were considered serious. Gastrointestinal AEs rarely resulted in dose limitation or treatment discontinuation. The most common grade 3 gastrointestinal AEs were nausea $(1.3 \%)$, vomiting $(1.0 \%)$, diarrhea $(0.8 \%)$, and constipation $(0.4 \%)$.

In practice, frequency and severity of gastrointestinal AEs vary according to the individual patient and concurrent medications. Prophylaxis with antinausea medications (eg, ondansetron) is suggested before treatment with carfilzomib, but these may also contribute to constipation. In addition, patients receiving opioids for pain may be more likely to experience gastrointestinal AEs. Dexamethasone, which is used prophylactically with administration of carfilzomib, also has antiemetic activity. In our experience, the majority of patients typically require no additional antinausea medication.

\section{Tumor lysis syndrome}

Tumor lysis syndrome is a metabolic complication resulting from the rapid destruction of cancer cells and release of their intracellular contents. ${ }^{34,35}$ The clinical manifestations of tumor lysis syndrome include increased lactate dehydrogenase, hyperuricemia, hyperphosphatemia, hypocalcemia, hyperkalemia, and renal failure. Patients with $\mathrm{MM}$ and a high tumor burden are considered to be at risk for tumor lysis syndrome.

In patients treated with carfilzomib, tumor lysis syndrome was observed in five patients in the Phase II trials, ${ }^{10,12}$ with three cases occurring prior to the inclusion of prophylaxis guidelines within study protocols. Following implementation of the prophylactic guidelines in the protocols, tumor lysis syndrome was only reported in one of 613 patients and thus is no longer considered to be a major concern.

To reduce the risk of tumor lysis syndrome, patients should be well hydrated before and after receiving carfilzomib therapy $(250-500 \mathrm{~mL}$ of intravenous fluid as indicated). ${ }^{10}$ Euvolemia should be maintained throughout treatment and monitored to prevent fluid overload. Blood chemistries should also be closely monitored. If tumor lysis syndrome occurs, therapy should be interrupted until symptoms resolve.

\section{Viral infections}

Herpes simplex infection or herpes zoster reactivation associated with MM is due to impaired lymphocyte function from the disease and/or treatment-related myelosuppression. 
In Phase II studies with carfilzomib, prophylactic oral antiviral therapy was recommended for all patients and required for patients with a history of herpes zoster or simplex in the 003 studies and all patients receiving subtherapeutic dexamethasone in the 005 study. ${ }^{12}$ Overall, $62.7 \%$ of patients received prophylactic therapy across the Phase II studies, and there was a low rate of herpes virus infection (25 patients, $4.8 \%$ ) with only one grade 3 event of "pain secondary to shingles". Antiviral prophylaxis is recommended prior to initiating carfilzomib for patients who have a history of herpes zoster infection. ${ }^{10}$

\section{Emory approach}

Based on our experience with carfilzomib throughout its clinical development, we have adapted its standard treatment protocol to meet the needs of our patients. We administer carfilzomib on Mondays and Tuesdays to ensure patients can easily access the clinic during the remainder of the working week, and have adopted a 30-minute infusion time for all doses of carfilzomib in all cycles, based on observations of lower AEs possibly attributed to carfilzomib, including dyspnea and fever. Additionally, we continue subtherapeutic dexamethasone prior to every infusion, again with potential benefits for prevention of infusion reactions, nausea, and drug-related fever, among other AEs. For patients in whom excessive hydration is a concern (eg, heart failure, renal dysfunction), we limit prehydration and post-hydration to $250 \mathrm{~mL}$ (500 $\mathrm{mL}$ total), and diurese as clinically indicated. We also initiate prophylactic oral acyclovir $400 \mathrm{mg}$ twice a day and double strength oral cotrimoxazole once daily on Monday, Wednesday, and Friday in patients who are not already receiving these agents because of therapy or disease-related risks. Our experience and practice is based on multiple prior and ongoing clinical trial patients as well as post-approval standard treatment in patients with relapsed/refractory disease.

\section{Summary}

Single-agent carfilzomib has an acceptable safety and tolerability profile in patients with RRMM, including those who are heavily pretreated or refractory to bortezomib and/or immunomodulatory drugs. In addition, carfilzomib has low rates of peripheral neuropathy, without cumulative or lateeffect toxicities. Carfilzomib provides a clinically significant advance in the treatment of RRMM.

\section{Acknowledgment}

Medical writing and editorial support were provided by Michael Raffin and Sabrina L Maurer, Fishawack
Communications, Inc., North Wales, PA, and funded by Onyx Pharmaceuticals, Inc., South San Francisco, CA, USA.

\section{Disclosure}

The author reports no conflicts of interest in this work.

\section{References}

1. Raab MS, Podar K, Breitkreutz I, Richardson PG, Anderson KC. Multiple myeloma. Lancet. 2009;374:324-339.

2. Augustson BM, Begum G, Dunn JA, et al. Early mortality after diagnosis of multiple myeloma: analysis of patients entered onto the United Kingdom Medical Research Council trials between 1980 and 2002 - Medical Research Council Adult Leukaemia Working Party. J Clin Oncol. 2005;23:9219-9226.

3. Nucci M, Anaissie E. Infections in patients with multiple myeloma in the era of high-dose therapy and novel agents. Clin Infect Dis. 2009;49: 1211-1225.

4. Lonial S, Mitsiades CS, Richardson PG. Treatment options for relapsed and refractory multiple myeloma. Clin Cancer Res. 2011;17: 1264-1277.

5. Laubach J, Mitsiades MCS, Mahindra A, et al. Management of relapsed and relapsed/refractory multiple myeloma. J Natl Compr Canc Netw. 2011;9:1209-1216.

6. Kumar SK, Rajkumar SV, Dispenzieri A, et al. Improved survival in multiple myeloma and the impact of novel therapies. Blood. 2008;111: 2516-2520.

7. Pingali SR, Haddad RY, Saad A. Current concepts of clinical management of multiple myeloma. Dis Mon. 2012;58:195-207.

8. Robin J, Fintel B, Pikovskaya O, Davidson C, Cilley J, Flaherty J. Multiple myeloma presenting with high-output heart failure and improving with anti-angiogenesis therapy: two case reports and a review of the literature. J Med Case Rep. 2008;2:229.

9. Chow AW, Lee CH, Hiwase DK, To LB, Horvath N. Relapsed multiple myeloma: who benefits from salvage autografts? Intern Med J. 2013;43:156-161.

10. Onyx Pharmaceuticals, Inc. Kyprolis ${ }^{\circledR}$ (carfilzomib) prescribing information. South San Francisco, CA, USA; Onyx Pharmaceuticals, Inc.; 2012.

11. Siegel DS, Martin T, Wang M, et al. A phase 2 study of single-agent carfilzomib (PX-171-003-A1) in patients with relapsed and refractory multiple myeloma. Blood. 2012;120:2817-2825.

12. Siegel D, Martin T, Nooka A, et al. Integrated safety profile of singleagent carfilzomib: experience from 526 patients enrolled in 4 phase 2 clinical studies. Haematologica. 2013;98:1753-1761.

13. Jagannath S, Vij R, Stewart AK, et al. An open-label single-arm pilot phase II study (PX-171-003-A0) of low-dose, single-agent carfilzomib in patients with relapsed and refractory multiple myeloma. Clin Lymphoma Myeloma Leuk. 2012;12:310-318.

14. Vij R, Siegel DS, Jagannath S, et al. An open-label, single-arm, phase 2 study of single-agent carfilzomib in patients with relapsed and/or refractory multiple myeloma who have been previously treated with bortezomib. Br J Haematol. 2012;158:739-748.

15. Vij R, Wang M, Kaufman JL, et al. An open-label, single-arm, phase 2 (PX-171-004) study of single-agent carfilzomib in bortezomib-naive patients with relapsed and/or refractory multiple myeloma. Blood. 2012;119:5661-5670.

16. Badros AZ, Vij R, Martin T, et al. Carfilzomib in multiple myeloma patients with renal impairment: pharmacokinetics and safety. Leukemia. 2013;27:1707-1714.

17. ClinicalTrials.gov. Phase 2 study of carfilzomib in relapsed and refractory multiple myeloma. Available from: http://clinicaltrials.gov/ show/NCT00511238. Accessed November 6, 2013.

18. ClinicalTrials.gov. Phase 2 study of carfilzomib in relapsed multiple myeloma. Available from: http://clinicaltrials.gov/show/NCT00530816. Accessed November 6, 2013. 
19. ClinicalTrials.gov. Study of the safety and pharmacokinetics of carfilzomib in subjects with relapsed and refractory multiple myeloma and varying degrees of renal function. Available from: http://clinicaltrials. gov/show/NCT00721734. Accessed November 6, 2013.

20. Nooka A, Badros A, Patel P, McCulloch L, Lonial S, Kaufman J. Hematologic safety data from four phase 2 studies of single-agent carfilzomib in relapsed and/or refractory multiple myeloma. Abstract (8086) and poster presented at the Annual Meeting of the American Society of Clinical Oncology; June 1-5, 2012, Chicago, IL, USA.

21. Common Terminology Criteria for Adverse Events version 3.0. Bethesda, MD, USA: US National Institute of Health, National Cancer Institute, Cancer Therapy Evaluation Program; 2006. Available from: http://ctep.cancer.gov/protocoldevelopment/electronic_applications/ docs/ctcae_index.pdf. Accessed March 12, 2014.

22. McBride L, Samuel C. The side effect profile of carfilzomib: from clinical trials to clinical practice. J Adv Pract Oncol. 2013;4:22-30.

23. Redic K. Carfilzomib: a novel agent for multiple myeloma. J Pharm Pharmacol. 2013;65:1095-1106.

24. Smith TJ, Khatcheressian J, Lyman GH, et al. 2006 update of recommendations for the use of white blood cell growth factors: an evidence-based clinical practice guideline. J Clin Oncol. 2006;24: 3187-3205.

25. Aapro MS, Bohlius J, Cameron DA, et al. 2010 update of EORTC guidelines for the use of granulocyte-colony stimulating factor to reduce the incidence of chemotherapy-induced febrile neutropenia in adult patients with lymphoproliferative disorders and solid tumours. Eur $J$ Cancer. 2011;47:8-32.

26. Crawford J, Armitage J, Balducci L, et al. Myeloid growth factors. J Natl Compr Canc Netw. 2013;11:1266-1290.

27. Armenian SH, Sun CL, Vase T, et al. Cardiovascular risk factors in hematopoietic cell transplantation survivors: role in development of subsequent cardiovascular disease. Blood. 2012;120:4505-4512.
28. Baker KS, Ness KK, Steinberger J, et al. Diabetes, hypertension, and cardiovascular events in survivors of hematopoietic cell transplantation: a report from the Bone Marrow Transplantation Survivor study. Blood. 2007;109:1765-1772.

29. Kistler KD, Rajangam K, Faich G, Lanes S. Cardiac event rates in patients with newly diagnosed and relapsed multiple myeloma in US clinical practice. Blood. 2012;120:Abstr 2916.

30. Yeh ET, Bickford CL. Cardiovascular complications of cancer therapy: incidence, pathogenesis, diagnosis, and management. J Am Coll Cardiol. 2009;53:2231-2247.

31. Lonial S, Niesvizky R, McCulloch L, Rajangam K, Vij R. Cardiac and pulmonary safety profile of single-agent carfilzomib from four phase 2 studies in patients with relapsed and/or refractory multiple myeloma. Abstract (4037) and poster presented at the Annual Meeting of the American Society of Hematology, December 8-11, 2012, Atlanta, GA, USA.

32. Harvey R, Lonial S, Wong A, McCulloch L, Badros A, Kaufman J. Renal safety profile of single-agent carfilzomib in patients with relapsed and/or refractory multiple myeloma from four phase 2 studies. Abstract and poster presented at Lymphoma and Myeloma Congress, October 25-27, 2012; New York, NY, USA

33. Martin T, Vij R, Badros AZ, Patel P, McCulloch L, Jagannath S. Carfilzomib is associated with a low rate of typically mild to moderate, non-dose limiting treatment-emergent peripheral neuropathy. Abstract 0857 and poster presented at Annual Meeting of the European Hematology Association, June 14-17, 2012, Amsterdam, The Netherlands.

34. Sezer O, Vesole DH, Singhal S, et al. Bortezomib-induced tumor lysis syndrome in multiple myeloma. Clin Lymphoma Myeloma. 2006; 7:233-235.

35. Howard SC, Jones DP, Pui CH. The tumor lysis syndrome. $N$ Engl J Med. 2011;364:1844-1854.
Clinical Pharmacology: Advances and Applications

\section{Publish your work in this journal}

Clinical Pharmacology: Advances and Applications is an international, peer-reviewed, open access journal publishing original research, reports, reviews and commentaries on all areas of drug experience in humans. The manuscript management system is completely online and includes a very quick and fair peer-review system, which is all easy to use.

\section{Dovepress}

Visit http://www.dovepress.com/testimonials.php to read real quotes from published authors. 